\title{
Detection Properties of a Neutron Counter Based on Scintillator, Wavelength Shifter, and Photomultiplier
}

\author{
R. Engels, Member, IEEE, G. Kemmerling, R. Cooper, and J. Schelten
}

\begin{abstract}
A simple neutron counter based on a scintillator strip, a wavelength shifter rod, and a photomultiplier was constructed in order to compare the properties of such a detector to a commercially available ${ }^{3} \mathrm{He}$ counter. A calculation of the number of photons per absorbed neutron shows that with the bright ${ }^{6} \mathrm{Li}^{158} \mathrm{Gd}\left({ }^{11} \mathrm{BO}_{3}\right)_{3}$ scintillator, light levels should be sufficient to perform pulse height discrimination. This has been experimentally demonstrated. Furthermore, the performance of this scintillation neutron counter has been measured in time-of-flight experiments where simultaneously the neutron spectra were recorded with a ${ }^{3} \mathrm{He}$ proportional counter.
\end{abstract}

Index Terms-Detector, neutron, photomultiplier, scintillator, wavelength shifter.

\section{INTRODUCTION}

$\mathbf{F}$ OR diffraction and inelastic scattering experiments at spallation sources, the well-known commercially available ${ }^{3}$ He proportional gas counters are not the best detector choice. With a ${ }^{3} \mathrm{He}$ counter one is not able to fulfill the three major detector requirements simultaneously:

1) sufficient detection efficiency for thermal and epithermal neutrons generated by inelastic scattering;

2) high temporal resolution for thermal and, in particular, for subthermal neutrons in time-of- flight (TOF) experiments;

3) high count-rate capability for detecting scattering processes with neutron bursts that are generated with pulsed sources.

For applications where these issues are important, a special scintillation detector with a detection area similar to that of a proportional counter might be useful as an alternative to the Helium 3 gas-filled detector tubes. We are not discussing that a scintillation counter with the liquid scintillator BC454 is not an alternative solution because the boron content is small and the hydrogen content is large.

In this paper, the design and the components of such a scintillation detector are described. It is based on a scintillator strip for neutron capture and a wavelength shifter to transport the

Manuscript received November 12, 2002; revised April 12, 2003. This work was funded by the SNS project via Argonne National Lab under the Contract OF-00827.

R. Engels and G. Kemmerling are with the Zentrallabor für Elektronik in the Forschungszentrum Juelich GmbH, 52425 Juelich, Germany (e-mail: r.engels@fz-juelich.de; g.kemmerling@fz-juelich.de).

R. Cooper is with the Oak Ridge National Laboratory, Oak Ridge, TN 37831-6474 USA (e-mail: cooperrg@sns.gov).

J. Schelten is with the Institut für Schicht- und Ionentechnik, Forschungszentrum Juelich GmbH, 52425 Juelich, Germany (e-mail: j.schelten@fz-juelich.de).

Digital Object Identifier 10.1109/TNS.2003.814546

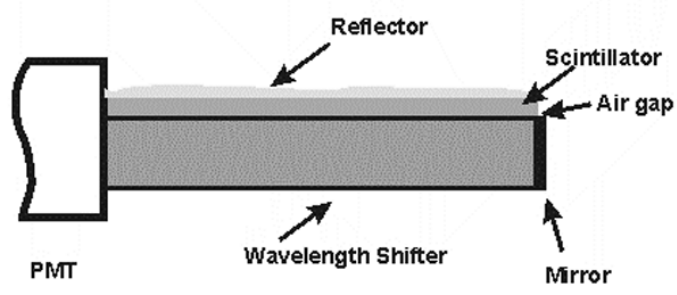

Fig. 1. Schematic view of the SWP counter of length $150 \mathrm{~mm}$ and cross section of $10 \mathrm{~mm} \times 10 \mathrm{~mm}$.

scintillation light to a photomultiplier. The properties of this scintillator, wavelength shifter, photomultiplier (SWP)-counter have been surveyed at the pulsed neutron source IPNS, Argonne National Laboratory (ANL). In TOF experiments, the temporal resolution for diffraction peaks has been measured. For comparison, data have been taken simultaneously with a ${ }^{3} \mathrm{He}$ proportional counter of similar detection area. The results of these measurements are described in detail.

\section{SWP COUNTER}

The commonly used ${ }^{3} \mathrm{He}$ counters have detection areas, typically 10-20 mm wide and 150-300 mm long. These detector dimensions are deliberately chosen for intensity reasons. Within the scattering plane one needs high spatial resolution and, therefore, the width must be small. In the direction perpendicular to the scattering plane one can tolerate a 15 times larger detector height in order to increase intensity without adversely affecting the spatial resolution.

For a scintillation counter with a thin LiGdBO scintillator strip of $10 \times 150 \mathrm{~mm}^{2}$ area, a wavelength shifter behind the strip is necessary in order to efficiently transport the scintillation light to a photomultiplier (PM). The PM is attached to one end of the wavelength shifter rod while a mirror covers the other end, as can be seen in Fig. 1.

In order to avoid attenuation of the wavelength shifted light due to absorption and scattering in the scintillator material, an air gap is maintained between the scintillator strip and the rod.

A fraction $\eta_{\text {ref }} \sim 75 \%$ of the $400 \mathrm{~nm}$ blue light generated by a neutron absorption event in the scintillator enters the rod and is converted with a quantum efficiency of $\eta_{\text {conv }} \sim 90 \%$ into green light.

Only the small fraction

$$
\eta_{\text {guide }}=0.5^{*}\left(1-\cos \left(\alpha_{c}\right)\right) \sim 0.17
$$

of this light is guided by the rod directly toward the PM, while the same small fraction is also reflected by the mirror to the 


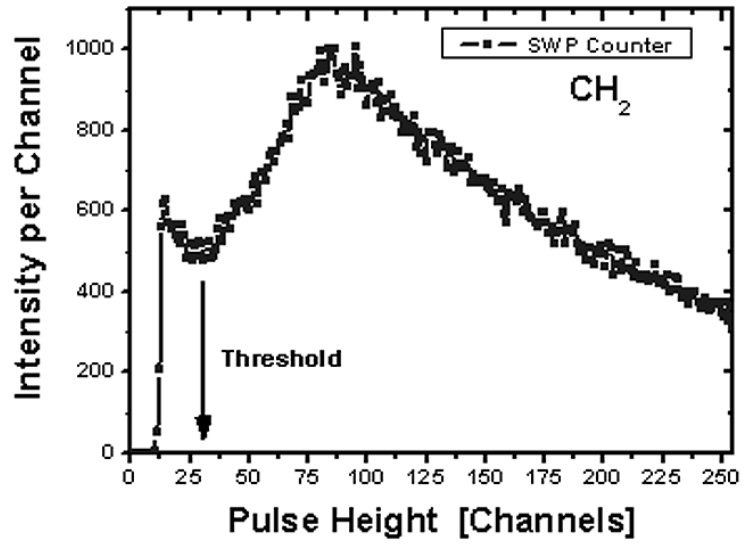

Fig. 2. Pulse height distribution measured of the SWP counter with $\mathrm{CH}_{2}$ sample.

PM. In (1) $\alpha_{c} \sim 50^{\circ}$ is the critical angle for total reflection. At the photocathode the quantum efficiency for converting photons into photoelectrons for green light $(g)$ is reduced by a factor $\eta_{e}(g) / \eta_{e}(b) \sim 0.5$ compared to the quantum efficiency of blue light $(b)$. Thus, the signal at the PM anode is reduced by a factor $\eta$ compared to the signal if all blue light were to hit the photo cathode. In this idealized case the reduction $\eta$ is given by

$$
\eta=\eta_{\text {ref }} \eta_{\text {conv }}\left(2 \eta_{\text {guide }}\right) \frac{\eta_{e}(g)}{\eta_{e}(b)} \sim 0.1 \text {. }
$$

For the new scintillator from photogenics-a crystalline powder of ${ }^{6} \mathrm{Li}{ }^{158} \mathrm{Gd}\left({ }^{11} \mathrm{BO}_{3}\right)_{3}$ [1] is mixed into a solidified polymer. The reason of choosing the three isotopes is described in [1]. The blue light generated per neutron absorption event is five times larger than the light generated in the well-known commercially available ${ }^{6} \mathrm{Li}$ glass scintillator. Thus, although there is a large reduction of light due to (2), the measured PM signal was still large enough for pulse height discrimination of the $\gamma$-background.

In general, the order of magnitude reduction of photoelectrons broadens the pulse height distribution and leads to less efficient gamma rejection by pulse height discrimination. For ${ }^{6} \mathrm{Li}$ glass scintillator this effect would be significant because the pulse height distribution is sharply peaked. However, for the ${ }^{6} \mathrm{Li}{ }^{158} \mathrm{Gd}\left({ }^{11} \mathrm{BO}_{3}\right)_{3}$ polymer mixture the additional broadening is not observable (Fig. 2), because of the inherent broad pulse height distribution due to energy deposition inhomogeneities and grain boundary effects.

Fortunately, this negligibly small additional broadening implies that the low gamma sensitivity measured with borate scintillator detectors should still be present in the SWP counter. In consequence of this discussion the discriminator level was set to the minimum of the pulse height spectrum as shown in Fig. 2. From an earlier comparison of a borate scintillator detector and of a ${ }^{3} \mathrm{He}$ proportional counter it can be concluded that both have the same low sensitivity of high-energy gammas [2].

\section{EXPERIMENTAL RESOLUTION}

The experimental arrangement for most of the TOF measurements is shown in Fig. 3.

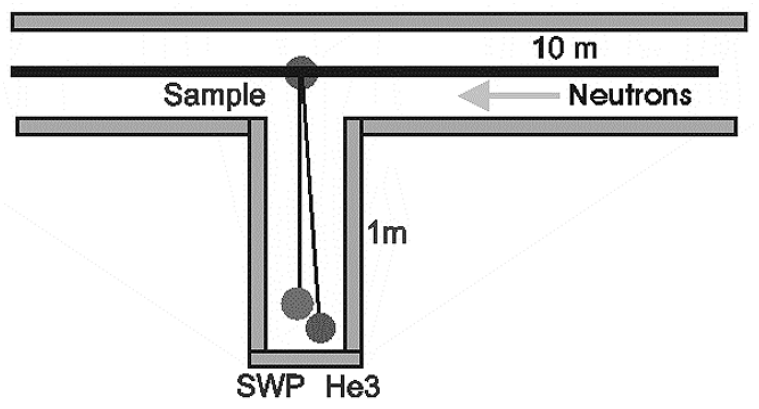

Fig. 3. Schematic drawing of the scattering geometry with the counters perpendicular to the scattering plane. The sample is located at a distance of about $20 \mathrm{~m}$ from the pulsed neutron source.

On the QUIP instrument at IPNS, the pulsed white neutron beam repeats with a frequency of $30 \mathrm{~Hz}$. At a distance of about $10 \mathrm{~m}$ from the neutron moderator, the samples are placed in the white beam. The scattered and diffracted neutron intensities are detected by two counters, the SWP scintillation counter and the ${ }^{3}$ He proportional counter at $\vartheta=90^{\circ}$ and $\vartheta+\Delta \vartheta$, respectively, with $\Delta \vartheta=1.7^{\circ}$. If a polycrystalline sample diffracts the beam, the angular difference leads to a shift of Bragg peaks according to the Bragg equations

$$
\begin{aligned}
2 d \sin \left(\frac{\vartheta}{2}\right) & =\lambda \\
\frac{\Delta \lambda}{\lambda} & =\frac{1}{\tan }\left(\frac{\vartheta}{2}\right) \Delta \vartheta=\frac{\Delta t}{t} .
\end{aligned}
$$

The width of the Bragg peaks are determined by the sum of various contributions:

$\delta t_{\text {source }}$ the finite temporal pulse width at the moderator surface. This contribution is wavelength dependent; however, it is only of the order of a few $\mu$ s.

$\delta t_{\text {path }}$ the timing uncertainty caused by the uncertainty in the flight path from the moderator surface via the sample to the position of absorption in the detector. This contribution is $\delta t_{\text {path }}=\delta L / v$ with

$\delta L=d_{\text {sample }}+d_{\text {detector }}$ determined by the thickness of the sample and by the depth of the detector, respectively, and with $v$ the neutron velocity.

$\delta t_{\text {Bragg }}$ the timing uncertainty caused by uncertainty in the diffraction angle due to the finite widths of sample and detector. This contribution is due to (4) $\delta t_{\mathrm{Bragg}}=$ $t / \tan (\vartheta / 2) \Delta \vartheta$ with $\Delta \vartheta=\left(d_{\text {sample }}+d_{\text {detector }}\right) /$ $L_{\text {sample-detector }}$.

With the geometrical parameters of Fig. 3 and for thermal neutrons with a velocity of $2000 \mathrm{~ms}^{-1}$ the broadening of a Bragg peak is $10 \mu \mathrm{s}$ and $160 \mu \mathrm{s}$ due the second and third effects, respectively.

\section{TOF MEASUREMENTS With VERTICAL COUNTERS}

Before beginning with the TOF experiments pulse height distributions were recorded in order to adjust the discrimination levels properly. Fig. 2 shows the pulse height distribution of the SWP counter and the cutoff where the discriminator level was set. The pulse height spectrum of the ${ }^{3} \mathrm{He}$ proportional counter is shown in Fig. 4. 


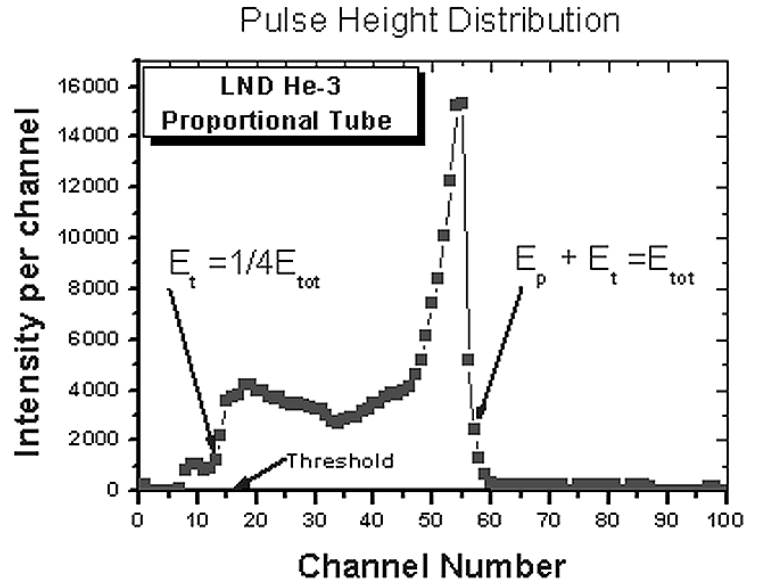

Fig. 4. Pulse height spectrum of the ${ }^{3} \mathrm{He}$ proportional counter. The characteristic features are caused by different wall effects for the generated triton and proton with low and high energy and with short and long traces, respectively. The threshold is set to the lower edge of the spectrum as indicated by an arrow.

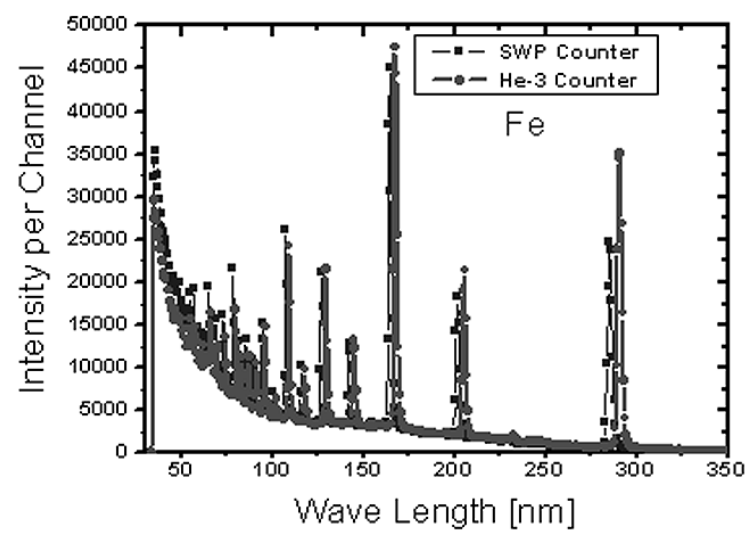

Fig. 5. TOF spectrum of polycrystalline iron as a plot of intensity.

With a polycrystalline Fe rod in the beam, strong and well-resolved diffraction peaks were observed, as shown in Fig. 5. There is a small shift of the Bragg peaks measured with the He counter with respect to those measured with the scintillation counter. The peak shifts are more pronounced at longer wavelengths. This is in agreement with the discussion of Section III and is caused by a slight difference in scattering angle for the two detectors.

The peaks are better separated in a diagram in which the measured intensity per time channel is plotted versus the momentum change $Q$ where $Q=4 \pi / \lambda \sin (\vartheta / 2)$. Such a diagram is shown in Fig. 6. The Bragg peaks are identified by their Miller indices. The measured $Q$ positions deviate from the calculated $2 \pi / d_{\mathrm{hkl}}$ by less than $1 \%$ regarding the correct but different scattering angles for the two detectors. The $d_{\mathrm{hkl}}$ values are lattice spacing with the Miller indices hkl.

Peak profiles are shown in Fig. 7. One observes essentially no difference between the peak pairs measured with different counters, which is in agreement with the predictions of Section III. The peak shift is about the FWHM of the peaks. Again, this is in agreement with the prediction of Section III.

Measurements with a polycrystalline $\mathrm{Al}$ rod leads to the similar results as observed with the iron sample. Fig. 8 shows the

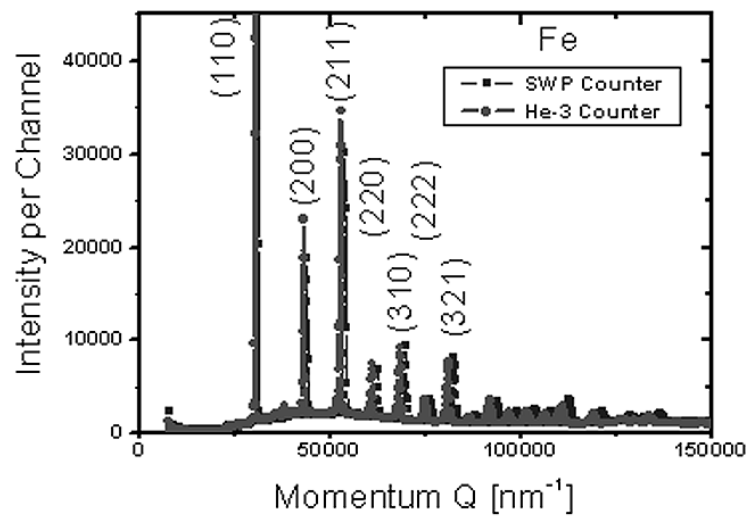

Fig. 6. Measured diffraction intensity versus neutron momentum transfer for six identified Fe Bragg peaks.

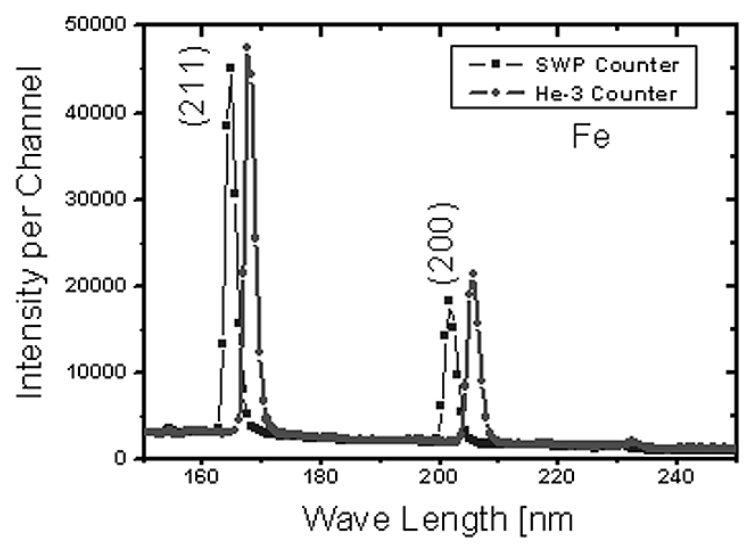

Fig. 7. Peak profiles as measured with the two counters.

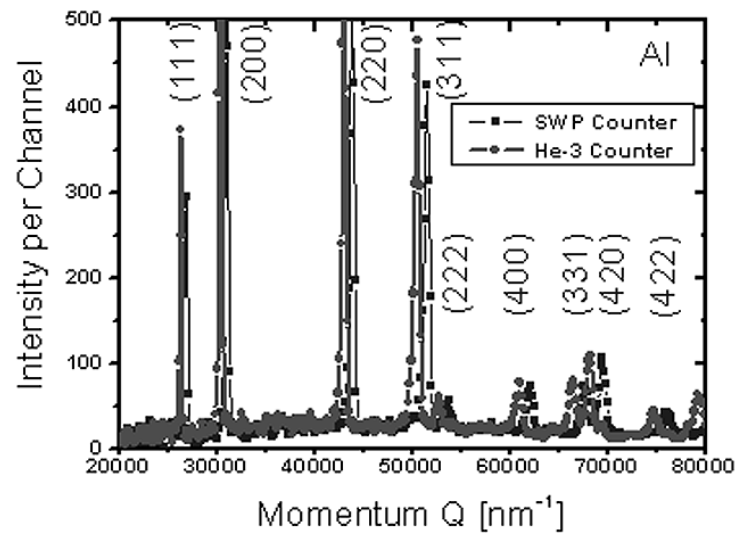

Fig. 8. Measured diffraction intensity versus neutron momentum transfer $Q$ for nine identified Al Bragg peaks.

diagram where the intensity is plotted versus the neutron momentum transfer $Q$ for nine identified Bragg peak.

With a 1-mm thick polyethylene strip sample, incoherently scattered neutrons were measured with both detectors. The scattered intensity is measured over five orders of magnitude within the wave length interval from 0.25 to 12 Angstroms (see Fig. 9). Both counters measure the same intensity because their sensitive detection area and their absorption probability are about the same. There is no observable systematic deviation, although 


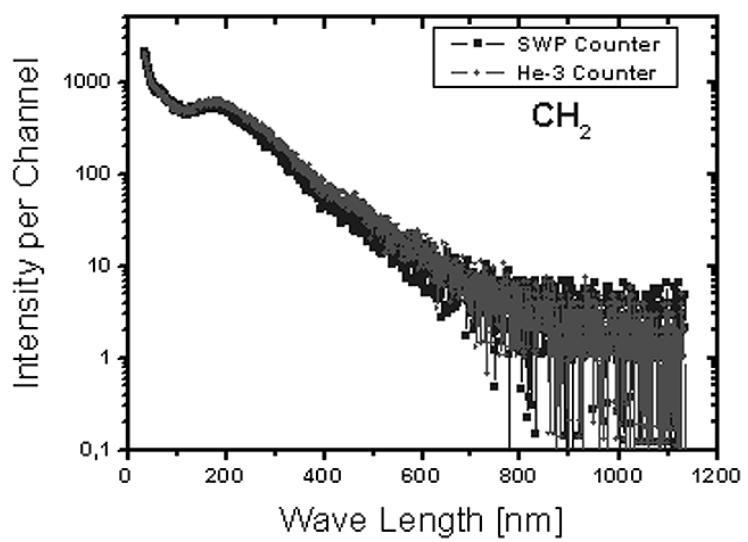

Fig. 9. Incoherently scattered neutron intensity as measured with the two counters versus the neutron wavelength in a logarithmic scale to cover five orders of magnitude.

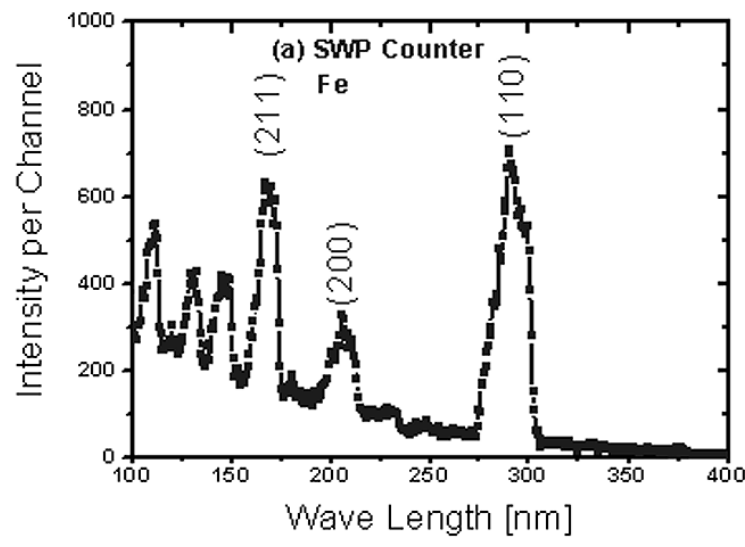

(a)

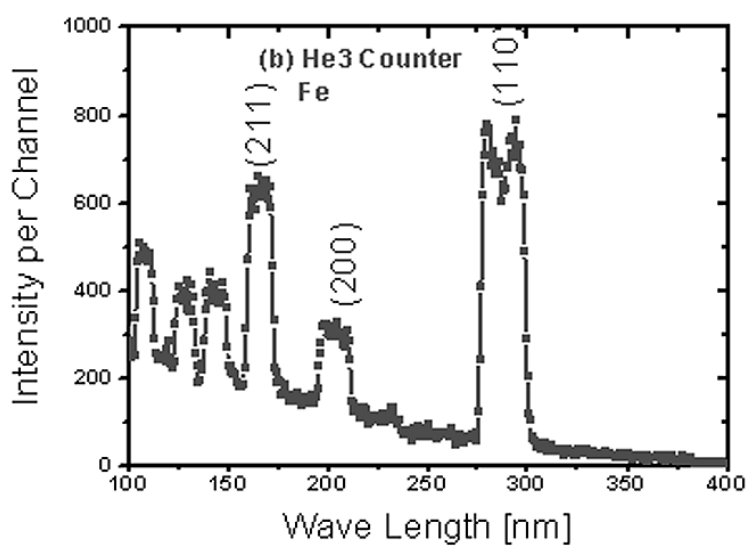

(b)

Fig. 10. Diffraction pattern of polycrystalline iron (a) with the detector axis within the scattering plane as measured with the ${ }^{3} \mathrm{He}$ proportional counter and (b) with the SWP scintillation counter in the lower and upper figures, respectively.

the relative fraction of gamma rays and background neutrons changes from very high to low along the curve.

\section{TOF MEASUREMENTS With HORIZONTAL COUNTERS}

In real diffraction and inelastic scattering experiments one uses the vertical geometry as described above. For a few measurements the long axis of the detectors was placed within the scattering plane. In this orientation, one expects very broad peaks that ideally have a rectangular shape. In TOF mode, the Bragg diffracted intensity scans across the detector. The scan begins with neutrons of shorter wave length at the smallest scattering angle, which interact at one detector end, and ends with neutrons of longer wave length and the largest scattering angle at the opposite detector end. Fig. 10 shows such diffraction patterns from two parallel detectors.

The data measured with the gas counter show the expected rectangular shape. When measured with the scintillation detector, the peak shapes are bowed. This is caused by a detector inhomogeneity along the detector axis. Probably the scintillator strips that were used have an inhomogeneous neutron response, which has been observed with prototype of Li Gd Borate scintillators

\section{SUMMARY}

The experimental result obtained with the SWP counter in comparison with a conventional ${ }^{3} \mathrm{He}$ counter demonstrate that

- both detectors perform identically concerning temporal resolution and detection efficiency;

- both counters have the same low gamma sensitivity as is known from the previous project report [1].

There is experimental evidence that the homogeneity of the SWP counter needs to be improved. This will happen when manufacturing techniques for this scintillator are refined.

Because the known light emission time of the SWP scintillator is much shorter than the charge collection time in a conventional ${ }^{3} \mathrm{He}$ counter, the SWP has superior rate handling capabilities, and is less affected by pileup effects.

Future experiments need to demonstrate that excellent temporal resolution and high detection efficiency can be achieved for epithermal neutrons. For these experiments, 3 to $10 \mathrm{~mm}$ thick transparent scintillator strips are required.

To build large area detectors with SWP counters a multidetector design is needed, which can be constructed economically. Light splitting and coincidence methods need to be developed in order to reduce the otherwise enormous equipment cost.

\section{ACKNOWLEDGMENT}

The authors would like to thank R. Porter, P. De Lurgio, and I. Naday, ANL, Argonne, IL, for their kind hospitality and for many valuable suggestions and interesting discussions. They would also like to thank W. Schäfer and E. Jansen, University of Bonn, Germany, for providing them with diffraction data and samples as well as for stimulating discussions.

\section{REFERENCES}

[1] J. B. Czirr, G. M. MacGillivray, R. R. MacGillivray, and P. J. Seddon, "Performance and characteristics of a new scintillator," Nucl. Instrum. Methods, vol. A 424, pp. 15-19, 1999.

[2] J. Schelten and R. Engels, "Experimental Comparison of a Neutron Scintillation Detector With a He Proportional Counter Internal Report, ANL,", 2000. 